Physics International 2 (1): 8-20, 2011

ISSN 1948-9803

(C) 2011 Science Publications

\title{
On the Closed Form Solutions of Linear and Nonlinear Cauchy Reaction-Diffusion Equations Using the Hybrid of Fourier Transform and Variational Iteration Method
}

\author{
${ }^{1}$ S.S. Nourazar, ${ }^{2}$ A. Nazari-Golshan and ${ }^{3}$ M. Nourazar \\ ${ }^{1}$ Department of Mechanical Engineering, \\ ${ }^{2}$ Department of Physics, \\ Amirkabir University of Technology, Tehran, Iran \\ ${ }^{3}$ Department of Physics, Azad Islamic University, Tehran, Iran
}

\begin{abstract}
Problem statement: In the present study, a hybrid of Fourier transform and Variational Iteration Method (FTVIM) is developed for solving the non-homogeneous linear and nonlinear partial differential equations of Cauchy reaction-diffusion problem. Approach: The closed form solutions obtained from the series solution of recursive sequences is valid for the entire range of problem domain. Results and Conclusion: Moreover, the very rapid convergence towards the exact solutions using the new method, FTVIM, indicates that the amount of computational work is much less than the computational work required for both the previous VIMs and the modified VIMs.
\end{abstract}

Key words: Cauchy reaction-diffusion, fourier transformation, variational iteration method, Nonhomogeneous, partial differential equation, exact solutions, restricted variational

\section{INTRODUCTION}

In the physical real-life phenomena, the majority of governing differential equations do not have analytic solutions. Moreover, due to the non-linear nature and variable coefficients of these differential equations, attentions are devoted to the approximate solutions obtained by semi analytical methods such as the Homotopy Perturbation Method (HPM) (He, 2009; Kasozi et al., 2011; Gupta and Singh, 2011; Othman et al., 2010; Gepreel, 2011; Aslanov, 2011) and Variational Iteration Method (VIM) (He et al., 2010; Shakeri, et al., 2009; Chen and Wang, 2010; Zhou and Yao, 2010; Odibat, 2010; Zhao and Xiao, 2010; Shang and Han, 2010; Turkyilmazoglu, 2011). The VIM is developed by employing a correction functional and a general Lagrange multiplier for the differential equation. Then by the variational principle the optimum value is obtained for the correction functional and it is solved using the iteration method (He et al., 2010; Shakeri, et al., 2009; Chen and Wang, 2010; Zhou and Yao, 2010; Odibat, 2010; Zhao and Xiao, 2010; Shang and Han, 2010; Turkyilmazoglu, 2011). The variational iteration method has been applied to so many engineering real-life problems so far (He et al., 2010; Shakeri et al., 2009; Chen and Wang, 2010; Zhou and Yao, 2010; Odibat, 2010; Zhao and Xiao, 2010; Shang and Han, 2010; Turkyilmazoglu, 2011). However, the solutions of these problems are valid only in one- directional problem domain either in time or space problem domain. In other words, the unsatisfied boundary conditions in the solutions of the VIM and other semi-analytical methods play no role in the final results (Kasozi et al., 2011; Gupta and Singh, 2011; Othman et al., 2010; Gepreel, 2011; Aslanov, 2011; Madani et al., 2011; He et al., 2010; Chen and Wang, 2010; Zhou and Yao, 2010; Odibat, 2010; Zhao and Xiao, 2010; Shang and Han, 2010; Turkyilmazoglu, 2011; Afshari et al., 2009; Aruchunan and Sulaiman, 2010). Therefore, there has been a deficiency as a built in short comes in the solutions using the VIM and other semi-analytical methods.

The basic objective of the present work is to develop a new modified VIM, the so called FTVIM, to overcome the deficiency caused due to validity of solution in small range of problem domain, because the boundary conditions are satisfied only in one dimension (He et al., 2010; Chen and Wang, 2010; Zhou and Yao, 2010; Odibat, 2010; Zhao and Xiao, 2010; Shang and Han, 2010; Turkyilmazoglu, 2011), when using the semi-analytical methods such as VIM. The new modified VIM is developed by combining the Fourier transform and VIM, where all conditions are satisfied over the entire range of time and space problem domains. It is shown mathematically that as the number of partial sum of the infinite series of approximate solution increases, very rapid convergence to the closed form solution, the exact solution of problem, is obtained

(C) 2011 S.S. Nourazar, A. Nazari-Golshan and M. Nourazar. This open access article is distributed under a Creative Commons Attribution (CC-BY) 3.0 license which permits unrestricted use, distribution, and reproduction in any medium, provided the original work is properly credited.

DOI: 10.3844/pisp.2011.8.20 
which is valid for the entire range of problem domain. In the present work, four different non-homogeneous linear and nonlinear partial differential equations, the Cauchy reaction-diffusion are solved using the new modified VIM (FTVIM). The closed form solutions for the four partial differential equations which are the exact solutions of the problems are obtained. Furthermore, the trends of very rapid convergence of the results toward the exact solutions have been demonstrated.

\section{MATERIALS AND METHODS}

Variational Iteration Method (VIM): The Variational Iteration Method (VIM) is originall y initiated by He et al. (2010). The basic idea of the VIM for solving nonlinear differential Eq. 1 is as follow; consider the following differential equation:

$\mathrm{E}(\mathrm{u})=\mathrm{g}(\mathrm{t})$

where, $\mathrm{E}$ is any differential operator. We construct a correction functional as follow Eq. 2 (Wazwaz, 2009):

$u_{n+1}=u_{n}+\int \lambda\left(L\left(u_{n}\right)+N\left(u_{n}\right)-g(t)\right) d t$

where, $\lambda$ is a general Lagrange multiplier that has to be optimized by the variational principle, $\mathrm{L}$ and $\mathrm{N}$ are linear and nonlinear operators and $\hat{\mathrm{u}}$ is the restricted variation of $u_{n}$ where, $\delta \hat{u}_{n}=0$ The main purpose of constructing the correction functional, Eq. 2, is the calculation of the optimal value of $\lambda$. Here for simplicity we assume that the operator $\mathrm{E}$ is linear. Thus the optimum value of $\lambda$ can be obtained by making use of the rule of integration by parts as Eq. 3:

$$
\int \lambda(\xi) u_{\mathrm{n}}^{\prime}(\xi) \mathrm{d} \xi=\lambda(\xi) \mathrm{u}_{\mathrm{n}}(\xi)-\int \lambda^{\prime}(\xi) \mathrm{u}_{\mathrm{n}}(\xi) \mathrm{d} \xi
$$

Equation 3 can be obtained by integration by parts. Having determined the Lagrange multiplier $\lambda(\xi)=-1$, the successive approximations $u_{n+1}, n \geq 0$ of the exact solution $\mathrm{u}$ is obtained upon choosing the proper value for $\mathrm{u}_{0}$ and $\mathrm{u}=\lim _{\mathrm{n} \rightarrow \infty} \mathrm{u}_{\mathrm{n}}$. As a matter of fact, the correction functional (Eq. 2) can provide several successive approximations and at the limit as u tends to infinity the resulting successive approximations approaches to the exact solution of the differential equation.

Basic Idea of FTVIM: The general forms of timedependent one-dimensional nonlinear partial differential equations are considered for illustrating the idea of the present method (FTVIM) as follows Eq. 4:
$E(u(x, t))=\varphi(x, t), x \geq 0, t \geq 0$

usually the operator E can be decomposed into two parts, linear and nonlinear, as:

$\mathrm{L}(\mathrm{u}(\mathrm{x}, \mathrm{t}))+\mathrm{N}(\mathrm{u}(\mathrm{x}, \mathrm{t}))=\varphi(\mathrm{x}, \mathrm{t})$

$u(x, 0)=f(x)$

and the boundary conditions Eq. 7:

$\mathrm{u}(0, \mathrm{t})=\mathrm{g}_{0}(\mathrm{t}), \mathrm{u}_{\mathrm{x}}(0, \mathrm{t})=\mathrm{g}_{1}(\mathrm{t})$

Applying Fourier transform to Eq. 5 and 6 we obtain as:

$\mathrm{F}\{\mathrm{L}(\mathrm{u}(\mathrm{x}, \mathrm{t}))\}+\mathrm{F}\{\mathrm{N}(\mathrm{u}(\mathrm{x}, \mathrm{t}))\}=\mathrm{F}\{\varphi(\mathrm{x}, \mathrm{t})\}$,

$\mathrm{F}\{\mathrm{u}(\mathrm{x}, \mathrm{t})\}=\mathrm{F}\{\mathrm{f}(\mathrm{x})\}$

where, $\mathrm{F}$ indicates the Fourier transform. We develop a correction functional to Eq. 8 as follow:

$$
\begin{aligned}
& \mathrm{F}\{\mathrm{u}(\mathrm{x}, \mathrm{t})\}_{\mathrm{n}+1}=\mathrm{F}\{\mathrm{u}(\mathrm{x}, \mathrm{t})\}_{\mathrm{n}}+ \\
& \int_{0}^{\mathrm{t}} \lambda(\xi)\left(\mathrm{F}\{\mathrm{L}(\mathrm{u}(\mathrm{x}, \xi))\}_{\mathrm{n}}+\mathrm{F}\{\mathrm{N}(\tilde{\mathrm{u}}(\mathrm{x}, \xi))\}_{\mathrm{n}}-\mathrm{F}\{\varphi(\mathrm{x}, \xi)\}\right) \mathrm{d} \xi
\end{aligned}
$$

where, $\tilde{\mathrm{u}}$ is a restricted variation and the first variation of it, $\tilde{\delta} \tilde{u}(x, \xi)$, vanishes. Taking the first variation from both sides of Eq. 9 we get the following:

$$
\begin{aligned}
& F\{u(x, t)\}_{n+1}=F\{u(x, t)\}_{n}+ \\
& \int_{0}^{t} \lambda(\xi)\left(F\{L(u(x, \xi))\}_{n}+F\{N(\tilde{u}(x, \xi))\}_{n}-F\{\varphi(x, \xi)\}\right) d \xi \\
& F\left\{\delta u_{n+1}(x, t)\right\}=F\left\{\delta u_{n}(x, t)\right\}+\int_{0}^{t} \lambda(\xi) F\left\{L\left(\delta u_{n}(x, \xi)\right)\right\} d \xi
\end{aligned}
$$

In Eq. 10 the Fourier transform is evaluated by integration by parts as follows:

$$
\begin{aligned}
& F\left\{\frac{\partial u(x, t)}{\partial x}\right\}=i \omega F\{u(x, t)\}-u(0, t) \\
& F\left\{\frac{\partial^{2} u(x, t)}{\partial x^{2}}\right\}=-\omega^{2} F\{u(x, t)\}-i \omega u(0, t)-u_{x}(0, t)
\end{aligned}
$$

$$
\mathrm{F}\left\{\frac{\partial \mathrm{u}(\mathrm{x}, \mathrm{t})}{\partial \mathrm{t}}\right\}=\frac{\partial}{\partial \mathrm{t}} \mathrm{F}\{\mathrm{u}(\mathrm{x}, \mathrm{t})\}
$$


Phy. Intl. 2 (1): 8-20, 2011

$\mathrm{F}\left\{\frac{\partial^{2} \mathrm{u}(\mathrm{x}, \mathrm{t})}{\partial \mathrm{t}^{2}}\right\}=\frac{\partial^{2}}{\partial \mathrm{t}^{2}} \mathrm{~F}\{\mathrm{u}(\mathrm{x}, \mathrm{t})\}$

and so on. The main step in the variational iteration method is to calculate the optimal value of $\lambda(\xi)$ in Eq. 10. Making use of Eq. 11-14 the optimal value of $\lambda(\xi)$ using Eq. 9 is obtained as follow:

$\hat{\mathrm{u}}_{\mathrm{n}+1}(\omega, \mathrm{t})=\hat{\mathrm{u}}_{\mathrm{n}}(\omega, \mathrm{t})$

$$
+\int_{0}^{t} \lambda(\xi 0)\left\{\begin{array}{l}
\left\{i \omega \hat{u}_{n}(\omega, \xi)-u(o, \xi)-\right. \\
\omega^{2} \hat{u}_{n}(\omega, \xi)-i \omega u(0, \xi)-u_{x}(0, \xi) \\
+F\{N(\hat{u}(x, \xi))\}_{n}-\hat{\varphi}(\omega, \xi) \\
\left.+\frac{\partial}{\partial \xi} \hat{u}_{n}(\omega, \xi)+\frac{\partial^{2}}{\partial \xi^{2}} \hat{u}_{n}(\omega, \xi)\right\}
\end{array}\right\} d \xi
$$

Superscripts denote the Fourier transform. Taking the first variations from Eq. 15 we get the following:

$$
\begin{aligned}
& \hat{\mathrm{u}}_{\mathrm{n}+1}(\omega, \mathrm{t})=\delta \hat{\mathrm{u}}_{\mathrm{n}}(\omega, \mathrm{t}) \\
& +\delta \int_{0}^{\mathrm{t} \lambda} \lambda(\xi)\left\{\begin{array}{l}
\left\{\mathrm{i} \omega \hat{\mathrm{u}}_{\mathrm{n}}(\omega, \xi)-\mathrm{u}(\mathrm{o}, \xi)-\right. \\
\omega^{2} \hat{\mathrm{u}}_{\mathrm{n}}(\omega, \xi)-\mathrm{i} \omega \mathrm{u}(0, \xi)-\mathrm{u}_{\mathrm{x}}(0, \xi) \\
+\mathrm{F}\{\mathrm{N}(\hat{\mathrm{u}}(\mathrm{x}, \xi))\}_{\mathrm{n}}-\hat{\varphi}(\omega, \xi)+\frac{\partial}{\partial \xi} \hat{\mathrm{u}}_{\mathrm{n}} \\
\left.(\omega, \xi)+\frac{\partial^{2}}{\partial \xi^{2}} \hat{\mathrm{u}}_{\mathrm{n}}(\omega, \xi)\right\}
\end{array}\right\} \mathrm{d} \xi
\end{aligned}
$$
as:

Taking integration by parts, Eq. 16 can be rewritten

$$
\begin{aligned}
& \delta \hat{\mathrm{u}}_{\mathrm{n}+1}(\omega, \mathrm{t})=\delta \hat{\mathrm{u}}_{\mathrm{n}}(\omega, \mathrm{t})+\lambda(\mathrm{t}) \delta \hat{\mathrm{u}}_{\mathrm{n}}(\omega, \mathrm{t})- \\
& \lambda(0) \delta \hat{\mathrm{u}}_{\mathrm{n}}(\omega, 0)-\frac{\partial}{\partial \mathrm{t}} \lambda(\mathrm{t}) \delta \hat{\mathrm{u}}_{\mathrm{n}}(\omega, \mathrm{t}) \\
& +\left.\frac{\partial}{\partial \mathrm{t}} \lambda(\mathrm{t})\right|_{\mathrm{t}=0} \delta \hat{\mathrm{u}}_{\mathrm{n}}(\omega, 0)+\left.\lambda(\xi)\right|_{\xi=\mathrm{t}} \\
& \frac{\partial}{\partial \mathrm{t}} \delta \hat{\mathrm{u}}_{\mathrm{n}}(\omega, \mathrm{t})-\left.\lambda(0) \frac{\partial}{\partial \mathrm{t}} \delta \hat{\mathrm{u}}_{\mathrm{n}}(\omega, \mathrm{t})\right|_{\mathrm{t}=0} \\
& +\int_{0}^{\mathrm{t}}\left\{\begin{array}{l}
\lambda(\xi)\left\{\mathrm{i} \omega \hat{\mathrm{u}}_{\mathrm{n}}(\omega, \xi)-\delta \mathrm{u}(\mathrm{o}, \xi)-\omega^{2} \delta \hat{\mathrm{u}}_{\mathrm{n}}(\omega, \xi)\right. \\
-\mathrm{i} \omega \mathrm{u}(0, \xi)-\delta \mathrm{u}_{\mathrm{x}}(0, \xi) \\
-\frac{\partial}{\partial \xi} \lambda(\xi) \delta \hat{\mathrm{u}}_{\mathrm{n}}(\omega, \xi)+\frac{\partial^{2}}{\partial \xi^{2}} \lambda(\xi) \delta \hat{\mathrm{u}}_{\mathrm{n}}(\omega, \xi)
\end{array}\right\} \mathrm{d} \xi
\end{aligned}
$$

Equation 17 yields the stationary conditions as:

$$
\begin{aligned}
& \left.\left\{1+\lambda(\mathrm{t})-\frac{\partial}{\partial \mathrm{t}} \lambda(\mathrm{t})\right\}\right|_{\xi=\mathrm{t}}=0 \\
& \left.\lambda(\xi)\right|_{\xi=\mathrm{t}}=0 \\
& \left.\left\{\left(-\omega^{2}+\mathrm{i} \omega\right) \lambda(\xi)-\frac{\partial}{\partial \xi} \lambda(\xi)+\frac{\partial^{2}}{\partial \xi^{2}} \lambda(\xi)\right\}\right|_{\xi=\mathrm{t}}=0
\end{aligned}
$$

Equation 18 in turn give the optimal value of $\lambda \mathrm{We}$ call this optimal value $\lambda_{\text {op }}$ substituting this back into Eq. 15 and by adding the nonlinear operator from Eq. 9 gives the iteration formula as:

$$
\begin{aligned}
& \hat{\mathrm{u}}_{\mathrm{n}+1}(\omega, \mathrm{t})=\hat{\mathrm{u}}_{\mathrm{n}}(\omega, \mathrm{t}) \\
& +\int \lambda_{\mathrm{op}}\left\{\begin{array}{l}
i \omega \hat{\mathrm{u}}_{\mathrm{n}}(\omega, \xi)-\mathrm{u}(0, \xi)-\omega^{2} \hat{\mathrm{u}}_{\mathrm{n}}(\omega, \xi) \\
-i \omega \mathrm{u}(0, \xi)-\mathrm{u}_{\mathrm{x}}(0, \xi)-\hat{\varphi}(\omega, \xi) \\
\left.+\frac{\partial}{\partial \xi} \hat{\mathrm{u}}_{\mathrm{n}}(\omega, \xi)+\frac{\partial^{2}}{\partial \xi^{2}} \hat{\mathrm{u}}_{\mathrm{n}}(\omega, \xi)+\mathrm{F}\{\mathrm{N}(\mathrm{x}, \xi))\right\}_{\mathrm{n}}
\end{array}\right\} \mathrm{d} \xi
\end{aligned}
$$

Using Eq. 19 the successive approximations $\hat{\mathrm{u}}_{\mathrm{n}+1}(\omega, \mathrm{t}), \mathrm{n} \geq 0$ can be obtained and the exact solution to the nonlinear partial differential equation is then equal to $\mathrm{u}=\lim _{\mathrm{n} \rightarrow \infty} \mathrm{u}_{\mathrm{n}}$. Equation 19 introduces the recursive relations as follows:

$$
\begin{aligned}
& \hat{\mathrm{u}}_{0}(\omega, \mathrm{t})=\hat{\mathrm{u}}(\omega, 0) \\
& \hat{\mathrm{u}}_{1}(\omega, \mathrm{t})=\hat{\mathrm{u}}_{0}(\omega, 0)
\end{aligned}
$$

$$
+\int_{0}^{\mathrm{t}} \lambda_{\mathrm{op}}\left\{\begin{array}{l}
\mathrm{i \omega \hat {u } _ { 0 }}(\omega, \xi)-\mathrm{u}_{0}(0, \xi)-\omega^{2} \hat{\mathrm{u}}_{0}(\omega, \xi) \\
-\mathrm{i} \omega \mathrm{u}_{0}(0, \xi)-\mathrm{u}_{\mathrm{x}}(0, \xi)-\hat{\varphi}(\omega, \xi) \\
\left.+\frac{\partial}{\partial \xi} \hat{\mathrm{u}}_{0}(\omega, \xi)+\frac{\partial^{2}}{\partial \xi^{2}} \hat{\mathrm{u}}_{0}(\omega, \xi)+\mathrm{F}\{\mathrm{N}(\mathrm{u}(\mathrm{x}, \xi))\}_{0}\right\}
\end{array}\right\} \mathrm{d} \xi
$$

$\hat{\mathrm{u}}_{2}(\omega, \mathrm{t})=\hat{\mathrm{u}}_{1}(\omega, 0)$

$$
+\int_{0}^{t} \lambda_{\mathrm{op}}(\xi)\left\{\begin{array}{l}
-\omega^{2} \hat{\mathrm{u}}_{1}(\omega, \xi)+\frac{\partial}{\partial \xi} \hat{\mathrm{u}}_{1}(\omega, \xi) \\
\left.+\frac{\partial^{2}}{\partial \xi^{2}} \hat{\mathrm{u}}_{1}(\omega, \xi)+\mathrm{F}\{\mathrm{N}(\mathrm{u}(\mathrm{x}, \xi))\}_{1}\right\}
\end{array}\right\} \mathrm{d} \xi
$$

$$
\begin{aligned}
& \hat{\mathrm{u}}_{3}(\omega, \mathrm{t})=\hat{\mathrm{u}}_{2}(\omega, \mathrm{t}) \\
& +\int_{0}^{\mathrm{t}} \lambda_{\mathrm{op}}(\xi)\left\{\begin{array}{l}
-\omega^{2} \hat{\mathrm{u}}_{2}(\omega, \xi)+\frac{\partial}{\partial \xi} \hat{\mathrm{u}}_{2}(\omega, \xi) \\
\left.+\frac{\partial^{2}}{\partial \xi^{2}} \hat{\mathrm{u}}_{2}(\omega, \xi)+\mathrm{F}\{\mathrm{N}(\mathrm{u}(\mathrm{x}, \xi))\}_{2}\right\}
\end{array}\right\} \mathrm{d} \xi
\end{aligned}
$$


And so on. Using the Maple package we solve Eq. 20-23 and then applying the inverse Fourier transform, that will define $\mathrm{u}_{0}, \mathrm{u}_{1}, \mathrm{u}_{2}, \mathrm{u}_{3}, \ldots, \mathrm{u}_{\mathrm{n}}$ and the solution is then equal to $u=\lim _{n \rightarrow \infty} u_{n}$. It is worth noting that Eq. 20-23 is a recursive approximation relations that have two advantages over the regular VIM. First: all initial and boundary conditions are satisfied. However, in the VIM either the initial conditions or the boundary conditions are satisfied. Usually the initial conditions are satisfied and the boundary conditions remain unsatisfied and any modifications to the unsatisfied boundary conditions do not affect the solution to the problem. Second: the amount of calculations is less than the one required for the VIM, because by taking the Fourier transform and integrating by parts the complexity of the method reduces considerably. Moreover the very rapid convergence to the exact solution can be achieved. In the following these advantages are shown by solving three nonhomogeneous linear partial differential equations and one more nonlinear partial differential equation of the Cauchy reaction-diffusion.

\section{RESULTS}

Fist we solve three linear partial differential equations and then we handle one more nonlinear partial differential equation. The Cauchy reaction-diffusion equation in one-dimensional and time-dependent case is written as (Akbarzade and Langari, 2011):

$$
\frac{\partial u}{\partial t}(x, t)=D \frac{\partial^{2}}{\partial x^{2}}(x, t)+r(x, t) u(x, t)
$$

where, $\mathrm{u}(\mathrm{x}, \mathrm{t})$ is the concentration of the substances, $r(x, t)$ is the reaction parameter at position $x$ and time $t$ and D is the diffusion coefficient. Equation 24 is solved subject to the following initial and boundary conditions Eq. 25 and 26:

$\mathrm{u}(\mathrm{x}, 0)=\mathrm{f}(\mathrm{x})$

$$
\mathrm{u}(0, \mathrm{t})=\mathrm{g}_{0}(\mathrm{t}), \frac{\partial}{\partial \mathrm{t}}(0, \mathrm{t})=\mathrm{g}_{2}(\mathrm{t})
$$

Example 1: The Kolmogorov-Petrovskii-Piskunov (KPP) is obtained by taking $\mathrm{D}=1, \mathrm{r}=-1$ in Eq. 24 as follow:

$$
\frac{\partial u}{\partial t}=D \frac{\partial^{2} u}{\partial x^{2}}-u, x \geq 0, t \geq 0
$$

$\mathrm{u}(\mathrm{x}, 0)=\mathrm{x}+\mathrm{e}^{-\mathrm{x}}$

$\mathrm{u}(0, \mathrm{t})=1, \mathrm{u}_{\mathrm{x}}(0, \mathrm{t})=\mathrm{e}^{-\mathrm{t}}-1$

The Fourier transform of Eq. 27 is Eq. 28:

$$
\begin{aligned}
& \frac{\partial \hat{\mathrm{u}}}{\partial \mathrm{t}}+\left(1+\omega^{2}\right) \hat{\mathrm{u}}+\mathrm{e}^{-\mathrm{t}}+i \omega-1=0 \\
& \hat{\mathrm{u}}(\omega, 0)=\frac{i(i+\omega \pi \delta(\omega))}{\omega^{2}}+\frac{1}{1+i \omega}
\end{aligned}
$$

where, $\delta(\omega)$ is the derivative of Dirac delta function. The Dirac delta function, $\delta(\omega-a)$ is defined as zero everywhere expect at $\omega=\mathrm{a}$ where it has a singularity.

We construct a correction functional as:

$$
\begin{aligned}
& \hat{\mathrm{u}}_{\mathrm{n}+1}(\omega, \mathrm{t})=\hat{\mathrm{u}}_{\mathrm{n}}(\omega, \mathrm{t})+ \\
& \int_{0}^{\mathrm{t}} \lambda(\xi)\left(\frac{\partial \hat{\mathrm{u}}_{\mathrm{n}}}{\partial \xi}+\left(1+\omega^{2}\right) \hat{\mathrm{u}}_{\mathrm{n}}+\mathrm{e}^{-\xi}+\mathrm{i} \omega-1\right) \mathrm{d} \xi
\end{aligned}
$$

Integrating by parts we get $\lambda(\xi)=-1$. Assuming $\hat{\mathrm{u}}_{0}(\omega, \mathrm{t})=\hat{\mathrm{u}}(\omega, 0)$ and substituting for the value of $\lambda(\xi)=-$ 1, using Eq. 29 the successive approximations $\hat{\mathrm{u}}_{\mathrm{n}+1}(\omega, \mathrm{t})$ are obtained as follows:

$$
\begin{aligned}
& \hat{\mathrm{u}}_{0}(\omega, \mathrm{t})=\frac{\mathrm{i}\left(\mathrm{i}+\omega^{2} \pi \delta(\omega)\right.}{\omega^{2}}+\frac{1}{1+\mathrm{i} \omega} \\
& \hat{\mathrm{u}}_{1}(\omega, \mathrm{t})=\hat{\mathrm{u}}_{0}(\omega, \mathrm{t})-\int_{0}^{\mathrm{t}}\left(\frac{\partial \hat{\mathrm{u}}_{0}}{\partial \xi}+\left(1+\omega^{2}\right) \hat{\mathrm{u}}_{0}+\mathrm{e}^{-\xi}+\mathrm{i} \omega-1\right) \mathrm{d} \xi \\
& \hat{\mathrm{u}}_{2}(\omega, \mathrm{t})=\hat{\mathrm{u}}_{1}(\omega, \mathrm{t})-\int_{0}^{\mathrm{t}}\left(\frac{\partial \hat{\mathrm{u}}_{1}}{\partial \xi}+\left(1+\omega^{2}\right) \hat{\mathrm{u}}_{1}+\mathrm{e}^{-\xi}+\mathrm{i} \omega-1\right) \mathrm{d} \xi \\
& \hat{\mathrm{u}}_{3}(\omega, \mathrm{t})=\hat{\mathrm{u}}_{2}(\omega, \mathrm{t})-\int_{0}^{\mathrm{t}}\left(\frac{\partial \hat{\mathrm{u}}_{2}}{\partial \xi}+\left(1+\omega^{2}\right) \hat{\mathrm{u}}_{1}+\mathrm{e}^{-\xi}+\mathrm{i} \omega-1\right) \mathrm{d} \xi \\
& \hat{\mathrm{u}}_{\mathrm{n}+1}(\omega, \mathrm{t})=\hat{\mathrm{u}}_{\mathrm{n}}(\omega, \mathrm{t})-\int_{0}^{\mathrm{t}}\left(\frac{\partial \hat{\mathrm{u}}_{\mathrm{n}}}{\partial \xi}+\left(1+\omega^{2}\right) \hat{\mathrm{u}}_{\mathrm{n}}+\mathrm{e}^{-\xi}+\mathrm{i} \omega-1\right) \mathrm{d} \xi
\end{aligned}
$$

By integrating the recursive approximate equations by parts, Eq. 30, we obtain the followings:

$$
\begin{aligned}
& \hat{\mathrm{u}}_{0}(\omega, \mathrm{t})=\frac{\mathrm{i}\left(\mathrm{i}+\omega^{2}(\pi \delta)\right)}{\omega^{2}}+\frac{1}{1+\mathrm{i} \omega} \\
& \hat{\mathrm{u}}_{1}(\omega, \mathrm{t})=\frac{1}{1+\mathrm{i} \omega}\left[1-\left(1+\omega^{2}\right) \mathrm{t}\right]+\left(\frac{\mathrm{i}\left(\mathrm{i}+\omega^{2}(\pi \delta)\right)}{\omega^{2}}\right) \\
& \left.\left[(1-\mathrm{t})-\omega^{2} \mathrm{t}\right)\right]-\frac{\mathrm{i} \omega \mathrm{t}^{2}}{2}+\left[\mathrm{e}^{-\mathrm{t}}-(1-\mathrm{t})\right]
\end{aligned}
$$


Phy. Intl. 2 (1): 8-20, 2011

$$
\begin{aligned}
& \hat{u}_{2}(\omega, t)=\frac{1}{1+i \omega}\left[1-\left(1+\omega^{2}\right) t+\left(1+\omega^{2}\right)^{2} \frac{t^{2}}{2}\right]+ \\
& \left.\left(\frac{i\left(i+\omega^{2}(\pi \delta)\right)}{\omega^{2}}\right)\left[(1-t)+\frac{t^{2}}{2}\right)-\omega^{2}\left(t-t^{2}\right)+\omega^{4} \frac{t^{2}}{2}\right] \\
& +i \omega\left(1+\omega^{2}\right) \frac{t^{3}}{6}-\frac{i \omega t^{2}}{2}+ \\
& \left.\left.\left[e^{-t}-(1-t)\right]-\left(1+\omega^{2}\right) \frac{t^{2}}{2}\right)\left[1-t+\frac{t^{2}}{2}-e^{-t}\right)\right] \\
& \hat{u}_{3}(\omega, t)=\frac{1}{1+i \omega}\left[1-\left(1+\omega^{2}\right) t+\left(1+\omega^{2}\right)^{2} \frac{t^{2}}{2}\right. \\
& \left.-\left(1+\omega^{2}\right)^{3} \frac{t^{3}}{6}\right]\left(\frac{i\left(i+\omega^{2}(\pi \delta)\right)}{\omega^{2}}\right)\left[\left(1-t+\frac{t^{2}}{2}-\frac{t^{3}}{6}\right)-\omega^{2}\left(t-t^{2}+\frac{t^{3}}{2}\right)\right. \\
& \left.+\omega^{4}\left(\frac{t^{2}}{2}-\frac{t^{3}}{2}\right]-\omega^{6} \frac{t^{3}}{6}\right]+i \omega\left(1+\omega^{2}\right)\left[\frac{t^{3}}{6}-\left(1+\omega^{2}\right) \frac{t^{4}}{24}\right]-\frac{i \omega t^{3}}{2} \\
& \left.+\left[e^{-t}-(1-t)\right]-\left(1+\omega^{2}\right)\left[1-t+\frac{t^{2}}{2}-e^{-t}\right)\right] \\
& -\left(1+\omega^{2}\right)^{2}\left[1-t+\frac{t^{2}}{2}-\frac{t^{3}}{6}-e^{-t}\right]
\end{aligned}
$$

And so on. The rest of the components were obtained using the Maple package. Using the Maple package the inverse Fourier transform of Eq. 31 is:

$$
\begin{aligned}
& u_{0}(x, t)=e^{-x}+x \\
& u_{1}(x, t)=e^{-x}+x(1-t) \\
& u_{2}(x, t)=e^{-x}+x\left(1-t+\frac{t^{2}}{2}\right) \\
& u_{3}(x, t)=e^{-x}+x\left(1-t+\frac{t^{2}}{2}-\frac{t^{3}}{6}\right)
\end{aligned}
$$

and so on. The Taylor series expansion for $\mathrm{e}^{-\mathrm{t}}$ is written as:

$$
\mathrm{e}^{-\mathrm{t}}=\sum_{\mathrm{n}=0}^{\infty} \frac{(-1)^{\mathrm{n}} \mathrm{t}^{\mathrm{n}}}{\mathrm{n} !}
$$

By substituting Eq. 33 into Eq. 32, thus Eq. 32 can ultimately be reduced to Eq. 34:

$$
u(x, t)=e^{-x}+x e^{-t}
$$

This is the exact solution of the problem, Eq. 27. In the following, Table 1 , the trend of convergence of the results of $u_{0}(x, t)$ to $u_{s}(x, t)$ of the FTVIM solution of
Eq. 27 towards the exact solution is shown. In Table 1 the percentage of relative errors of the results of $u_{0}(x, t)$ to $\mathrm{u}_{\mathrm{s}}(\mathrm{x}, \mathrm{t})$ of the FTVIM solution of Eq. 27 at each location along the $\mathrm{x}$ axis and at different times are shown. The trend of very rapid convergence, the maximum relative error of less than $0.03 \%$, of the results towards the exact solution is clearly shown.

Example 2: The Kolmogorov-Petrovskii-Piskunov (KPP) is obtained by taking $\mathrm{D}=1, \mathrm{r}=2 \mathrm{t}$ in Eq. 24 as follow:

$\frac{\partial u}{\partial t}=\frac{\partial^{2} u}{\partial x^{2}}+2 t u, x \geq 0, t \geq 0$

$\mathrm{u}(\mathrm{x}, 0)=\mathrm{e}^{-\mathrm{x}}$

$u(0, t)=e^{t+t^{2}}, u_{x}(0, t)=e^{t+t^{2}}$

The Fourier transform of Eq. 35 is Eq. 36:

$\frac{\partial \hat{u}}{\partial t}+\left(\omega^{2}-2 t\right) \hat{u}+e^{t+t^{2}}(i \omega+1)=0$

$\hat{\mathrm{u}}(\omega, 0)=\frac{1}{1+\mathrm{i} \omega}$

We construct a correction functional as:

$\hat{\mathrm{u}}_{\mathrm{n}+1}(\omega, \mathrm{t})=\hat{\mathrm{u}}_{\mathrm{n}}(\omega, \mathrm{t})$

$+\int_{0}^{\mathrm{t}} \lambda(\xi)\left(\frac{\partial \hat{\mathrm{u}}}{\partial \xi}+\left(\omega^{2}-2 \xi\right) \hat{\mathrm{u}}_{\mathrm{n}}\right.$

$+\mathrm{e}^{\xi+\xi^{2}}(\mathrm{i} \omega+1) \mathrm{d} \xi$

Integrating by parts we get $\lambda(\xi)=-1$. Assuming $\hat{\mathrm{u}}_{0}(\omega, \mathrm{t})=\hat{\mathrm{u}}(\omega, 0)$ and substituting for the value of $\lambda(\xi)=-$ 1, Eq. 37 can be rewritten as:

$$
\begin{aligned}
& \hat{\mathrm{u}}_{0}(\omega, \mathrm{t})=\frac{1}{1+\mathrm{i} \omega} \\
& \hat{\mathrm{u}}_{1}(\omega, \mathrm{t})=\hat{\mathrm{u}}_{0}(\omega, \mathrm{t})-\int_{0}^{\mathrm{t}} \frac{\partial \hat{\mathrm{u}}}{\partial \xi}+\left(\omega^{2}-2 \xi\right) \hat{\mathrm{u}}_{0}+\mathrm{e}^{\xi+\xi^{2}}(\mathrm{i} \omega+1) \mathrm{d} \xi \\
& \hat{\mathrm{u}}_{2}(\omega, \mathrm{t})=\hat{\mathrm{u}}_{1}(\omega, \mathrm{t})- \\
& \int_{0}^{\mathrm{t}} \frac{\partial \hat{\mathrm{u}}_{1}}{\partial \xi}+\left(\omega^{2}-2 \xi\right) \hat{\mathrm{u}}_{1}+\mathrm{e}^{\xi+\xi^{2}}(\mathrm{i} \omega+1) \mathrm{d} \xi
\end{aligned}
$$


Phy. Intl. 2 (1): 8-20, 2011

Table 1: Shows the percentage of relative errors of the results of $u_{0}(x, t)$ to $u_{s}(x, t)$ of the FTVIM solution of Eq. 27

\begin{tabular}{|c|c|c|c|c|}
\hline & & \multicolumn{3}{|c|}{ Percentage of relative error (\%RE) } \\
\hline & & $\mathrm{x}=1$ & $\mathrm{x}=1.5$ & $x=2$ \\
\hline \multirow[t]{6}{*}{$\mathrm{t}=0.1$} & $\mathrm{u}_{0}(\mathrm{x}, \mathrm{t})$ & 0.07477121192 & 0.09032214097 & 0.0978530456 \\
\hline & $\mathrm{u}_{1}(\mathrm{x}, \mathrm{t})$ & 0.003800859527 & 0.004591362922 & 0.004974182863 \\
\hline & $\mathrm{u}_{2}(\mathrm{x}, \mathrm{t})$ & 0.0001277440452 & 0.00015431227 & 0.000167178564 \\
\hline & $\mathrm{u}_{3}(\mathrm{x}, \mathrm{t})$ & 0.000003209433 & 0.000003876900 & 0.0000041999781 \\
\hline & $\mathrm{u}_{4}(\mathrm{x}, \mathrm{t})$ & $6.467320576 \mathrm{e}-8$ & 7.78290731 e-8 & 8.431832740 e- 8 \\
\hline & $\mathrm{u}_{\mathrm{s}}(\mathrm{x}, \mathrm{t})$ & 8.46122714 e-10 & 1.26551338 e-9 & $1.542408428 \mathrm{e}-9$ \\
\hline \multirow[t]{6}{*}{$\mathrm{t}=0.4$} & $\mathrm{u}_{0}(\mathrm{x}, \mathrm{t})$ & 0.3175497177 & 0.4025035111 & 0.4467282577 \\
\hline & $\mathrm{u}_{1}(\mathrm{x}, \mathrm{t})$ & 0.0677326919 & 0.08585315872 & 0.09528620484 \\
\hline & $\mathrm{u}_{2}(\mathrm{x}, \mathrm{t})$ & 0.0093237900 & 0.01181817525 & 0.0131166876 \\
\hline & $\mathrm{u}_{3}(\mathrm{x}, \mathrm{t})$ & 0.00095040761 & 0.001204669280 & 0.0013370311 \\
\hline & $\mathrm{u}_{4}(\mathrm{x}, \mathrm{t})$ & 0.000077012174 & 0.00009761517 & 0.0001083405 \\
\hline & $\mathrm{u}_{\mathrm{s}}(\mathrm{x}, \mathrm{t})$ & 0.000005181374 & 0.00000656758 & 0.000007289417 \\
\hline \multirow[t]{6}{*}{$\mathrm{t}=0.7$} & $\mathrm{u}_{0}(\mathrm{x}, \mathrm{t})$ & 0.5823426568 & 0.7800782162 & 0.8921791200 \\
\hline & $\mathrm{u}_{1}(\mathrm{x}, \mathrm{t})$ & 0.2274069648 & 0.3046234333 & 0.3483992516 \\
\hline & $\mathrm{u}_{2}(\mathrm{x}, \mathrm{t})$ & 0.0560054027 & 0.0750221439 & 0.08580317854 \\
\hline & $\mathrm{u}_{3}(\mathrm{x}, \mathrm{t})$ & 0.0101241497 & 0.0135618240 & 0.01551072187 \\
\hline & $\mathrm{u}_{4}(\mathrm{x}, \mathrm{t})$ & 0.0014485219 & 0.00194037040 & 0.002219210747 \\
\hline & $\mathrm{u}_{\mathrm{s}}(\mathrm{x}, \mathrm{t})$ & 0.00017165199 & 0.00022993681 & 0.000262979752 \\
\hline
\end{tabular}

$$
\begin{aligned}
& \hat{\mathrm{u}}_{3}(\omega, \mathrm{t})=\hat{\mathrm{u}}_{2}(\omega, \mathrm{t})-\int_{0}^{\mathrm{t}} \frac{\partial \hat{\mathrm{u}}_{2}}{\partial \xi}+\left(\omega^{2}-2 \xi\right) \hat{\mathrm{u}}_{2}+\mathrm{e}^{\xi+\xi^{2}}(\mathrm{i} \omega+1) \mathrm{d} \xi \\
& \hat{\mathrm{u}}_{\mathrm{n}+1}(\omega, \mathrm{t})=\hat{\mathrm{u}}_{\mathrm{n}}(\omega, \mathrm{t})-\int_{0}^{\mathrm{t}} \frac{\partial \hat{\mathrm{u}}_{\mathrm{n}}}{\partial \xi}+\left(\omega^{2}-2 \xi\right) \hat{\mathrm{u}}_{\mathrm{n}}+\mathrm{e}^{\xi+\xi^{2}}(\mathrm{i} \omega+1) \mathrm{d} \xi
\end{aligned}
$$

And so on by integrating the recursive approximate equations by parts, Eq. 38, we obtain the followings Eq. 39:

$$
\begin{aligned}
& \hat{\mathrm{u}}_{0}(\omega, \mathrm{t})=\frac{1}{1+\mathrm{i} \omega} \\
& \hat{\mathrm{u}}_{1}=\frac{1}{1+\mathrm{i} \omega}\left(1+\mathrm{t}^{2}-\omega \mathrm{t}\right)(1+\mathrm{i} \omega) \\
& \left(\frac{\mathrm{i} \sqrt{\pi}}{2}\right) \mathrm{e}^{-\frac{1}{4}}\left(\operatorname{erf}\left(\mathrm{it}+\frac{\mathrm{i}}{2}\right)-\operatorname{erf}\left(\frac{\mathrm{i}}{2}\right)\right) \\
& \left.\hat{\mathrm{u}}_{2}=\frac{1}{1+\mathrm{i} \omega}\left(1+\mathrm{t}^{2}+\frac{\mathrm{t}^{4}}{2}-\omega^{2}\right)\left(\mathrm{t}+\mathrm{t}^{3}\right)+\omega^{2}\right) \\
& \left.\left(\mathrm{t}+\mathrm{t}^{3}\right)+\omega^{4} \frac{\mathrm{t}^{2}}{2}\right)+(1+\mathrm{i} \omega) \\
& {\left[\mathrm{a}_{21}\right]-\omega^{2}(1+\mathrm{i} \omega)\left[\mathrm{a}_{22}\right]+(1+\mathrm{i} \omega)} \\
& \left(\frac{i \sqrt{\pi}}{2}\right) e^{-\frac{1}{4}}\left(\operatorname{erf}\left(i t+\frac{i}{2}\right)-\operatorname{erf}\left(\frac{i}{2}\right)\right) \\
& \mathrm{a}_{21}=-\frac{1}{4}+\frac{1}{4} \mathrm{e}^{\mathrm{t}+\mathrm{t}^{2}}-\frac{1}{2} \mathrm{te}^{\mathrm{t}+\mathrm{t}^{2}} \\
& +\left(\frac{\mathrm{i} \sqrt{\pi}}{8}\right) \mathrm{e}^{-\frac{1}{4}} \operatorname{erf}\left(\frac{\mathrm{i}}{2}\right)+4 \mathrm{t}^{2} \operatorname{erf}\left(\frac{\mathrm{i}}{2}\right) \\
& -4 t^{2} \operatorname{erf}\left(\frac{i}{2}(1+2 t)\right)-\operatorname{erf}\left(\frac{i}{2}(1+2 t)\right)
\end{aligned}
$$

$$
\begin{aligned}
& \mathrm{a}_{22}=-\frac{1}{2}+\frac{1}{2} \mathrm{e}^{1+\mathrm{t}^{2}} \\
& +\left(\frac{\mathrm{i} \sqrt{\pi}}{2}\right) \mathrm{e}^{-\frac{1}{4}}\left(-\frac{1}{2} \operatorname{erf}\left(\frac{\mathrm{i}}{2}\right)+-\frac{1}{2} \operatorname{erf}\left(\frac{\mathrm{i}}{2}(1+2 \mathrm{t})\right.\right. \\
& \left.+t \operatorname{erf}\left(\frac{i}{2}(1+2 t)\right)-\operatorname{erf}\left(\frac{i}{2}\right)\right) \\
& \hat{\mathrm{u}}_{3}=\frac{1}{1+\mathrm{i} \omega}\left(1+\mathrm{t}^{2}+\frac{\mathrm{t}^{4}}{2}+\frac{\mathrm{t}^{6}}{6}-\omega^{2}\right) \\
& \left.\left(\mathrm{t}+\mathrm{t}^{3}+\frac{\mathrm{t}^{3}}{2}\right)+\omega^{4}\left(\frac{\mathrm{t}^{2}}{2}+\frac{\mathrm{t}^{4}}{6}\right)-\omega^{6} \frac{\mathrm{t}^{3}}{6}\right) \\
& +\frac{1}{16} \mathrm{e}^{\frac{1}{4}(1+2 t)^{2}} \omega^{4}(1+\mathrm{i} \omega)\left[\mathrm{a}_{31}\right]++\frac{1}{48} \mathrm{e}^{\frac{1}{4}(1+2 \mathrm{t})^{2}} \\
& \omega^{2}(1+i \omega)\left[a_{32}\right]-\frac{1}{24} e^{\frac{1}{4}(1+2 t)^{2}} \omega^{2}(1+i \omega)\left[a_{33}\right] \\
& +\frac{1}{64}(1+\mathrm{i} \omega) \mathrm{e}^{\frac{1}{4}(1+2 t)^{2}}\left[\mathrm{a}_{34}\right]+\omega^{2}\left[\mathrm{a}_{35}\right]-\left[\mathrm{a}_{36}\right]- \\
& (1+i \omega)\left(\frac{i \sqrt{\pi}}{2}\right) e^{-\frac{1}{4}}\left(\operatorname{erf}\left(i t+\frac{i}{2}\right)-\operatorname{erf}\left(\frac{i}{2}\right)\right) \\
& a_{31}=-2 e^{-\frac{1}{4}}(1+2 t)^{2}-8 t e^{-\frac{1}{4}(1+2 t)^{2}}+2 e^{-\frac{1}{4}}+4 e^{-\frac{1}{4}} \\
& +\mathrm{i} \sqrt{\pi}\left(\mathrm{e}^{-\frac{1}{2}-\mathrm{t}-\mathrm{t}^{2}} \operatorname{erf}\left(\frac{\mathrm{i}}{2}\right)-4 \mathrm{t} \mathrm{e}^{-\frac{1}{2}-\mathrm{t}-\mathrm{t}^{2}}\right. \\
& \operatorname{erf}\left(\frac{i}{2}\right)+4 t e^{-\frac{1}{2}-t-t^{2}} \operatorname{erf}\left(i t+\frac{i}{2}\right) \\
& -\mathrm{e}^{-\frac{1}{2}-\mathrm{t}-\mathrm{t}^{2}} \operatorname{erf}\left(\mathrm{it}+\frac{\mathrm{i}}{2}\right)+4 \mathrm{t}^{2} \mathrm{e}^{-\frac{1}{2}-\mathrm{t}-\mathrm{t}^{2}} \\
& \left.\operatorname{erf}\left(i t+\frac{i}{2}\right)-4 t^{2} e^{-\frac{1}{2}-t-t^{2}} \operatorname{erf}\left(\frac{i}{2}\right)\right)
\end{aligned}
$$


Phy. Intl. 2 (1): 8-20, 2011

$$
\begin{aligned}
& a_{32}=6 e^{-\frac{1}{4}}(1+2 t)^{2}+24 t^{2} e^{-\frac{1}{4}(1+2 t)^{2}}-6 e^{-\frac{1}{4}}-4 t e^{-\frac{1}{4}}-16 t^{2} e^{-\frac{1}{4}} \\
& +\mathrm{i} \sqrt{\pi}\left(5 \mathrm{e}^{-\frac{1}{2}-\mathrm{t}-\mathrm{t}^{2}} \operatorname{erf}\left(\frac{\mathrm{i}}{2}\right)+12 \mathrm{t}^{2} \mathrm{e}^{-\frac{1}{2}-\mathrm{t}-\mathrm{t}^{2}}\right. \\
& \operatorname{erf}\left(\frac{\mathrm{i}}{2}\right)-5 \mathrm{e}^{-\frac{1}{2}-\mathrm{t}-\mathrm{t}^{2}} \operatorname{erf}\left(\mathrm{it}+\frac{\mathrm{i}}{2}\right) \\
& -12 \mathrm{t}^{2} \mathrm{e}^{-\frac{1}{2}-\mathrm{t}-\mathrm{t}^{2}} \operatorname{erf}\left(\mathrm{it}+\frac{\mathrm{i}}{2}\right)-16 \mathrm{t}^{3} \mathrm{e}^{-\frac{1}{2}-\mathrm{t}-\mathrm{t}^{2}} \\
& \left.\operatorname{erf}\left(i t+\frac{i}{2}\right)+16 t^{3} e^{-\frac{1}{2}-t-t^{2}} \operatorname{erf}\left(\frac{i}{2}\right)\right) \\
& a_{33}=6 e^{-\frac{1}{4}(1+2 t)^{2}}-6 t e^{-\frac{1}{4}(1+2 t)^{2}}-6 e^{-\frac{1}{4}}+2 t e^{-\frac{1}{4}}-4 t^{2} e^{-\frac{1}{4}} \\
& +\mathrm{i} \sqrt{\pi}\left(5 \mathrm{e}^{-\frac{1}{2}-\mathrm{t}-\mathrm{t}^{2}} \operatorname{erf}\left(\frac{\mathrm{i}}{2}\right)+3 \mathrm{te} \mathrm{e}^{-\frac{1}{2}-\mathrm{t}-\mathrm{t}^{2}}\right. \\
& \operatorname{erf}\left(\frac{i}{2}\right)+4 t^{3} e^{-\frac{1}{2}-t-t^{2}} \operatorname{erf}\left(i t+\frac{i}{2}\right) \\
& -5 \mathrm{e}^{-\frac{1}{2}-\mathrm{t}-\mathrm{t}^{2}} \operatorname{erf}\left(\mathrm{it}+\frac{\mathrm{i}}{2}\right)-4 \mathrm{t}^{3} \mathrm{e}^{-\frac{1}{2}-\mathrm{t}-\mathrm{t}^{2}} \\
& \operatorname{erf}\left(i t+\frac{i}{2}\right)-3 t e^{-\frac{1}{2}-t-t^{2}} \operatorname{erf}\left(i t+\frac{i}{2}\right) \\
& a_{34}=-18 e^{-\frac{1}{4}(1+2 t)^{2}}-16 t^{2} e^{-\frac{1}{4}(1+2 t)^{2}}+18 e^{-\frac{1}{4}} \\
& -20 \mathrm{te}^{-\frac{1}{4}}+8 \mathrm{t}^{2} \mathrm{e}^{-\frac{1}{4}}-16 \mathrm{t}^{3} \mathrm{e}^{-\frac{1}{4}} \\
& +\mathrm{i} \sqrt{\pi}\left(8 \mathrm{t}^{2} \mathrm{e}^{-\frac{1}{2}-\mathrm{t}-\mathrm{t}^{2}} \operatorname{erf}\left(\frac{\mathrm{i}}{2}\right)-16 \mathrm{t}^{4} \mathrm{e}^{-\frac{1}{2}-\mathrm{t}-\mathrm{t}^{2}} \operatorname{erf}\left(\mathrm{it}+\frac{\mathrm{i}}{2}\right)\right. \\
& +16 \mathrm{t}^{4} \mathrm{e}^{-\frac{1}{2}-\mathrm{t}-\mathrm{t}^{2}} \operatorname{erf}\left(\frac{\mathrm{i}}{2}\right)+\mathrm{e}^{-\frac{1}{2}-\mathrm{t}-\mathrm{t}^{2}} \operatorname{erf}\left(\frac{\mathrm{i}}{2}\right) \\
& -8 \mathrm{t}^{2} \mathrm{e}^{-\frac{1}{2}-\mathrm{t}-\mathrm{t}^{2}} \operatorname{erf}\left(\mathrm{it}+\frac{\mathrm{i}}{2}\right)-4 \mathrm{e}^{-\frac{1}{2}-\mathrm{t}-\mathrm{t}^{2}} \operatorname{erf}\left(\mathrm{it}+\frac{\mathrm{i}}{2}\right) \\
& \mathrm{a}_{35}=-\frac{1}{2}+\frac{1}{2} \mathrm{e}^{\mathrm{t}+\mathrm{t}^{2}} \\
& +\left(\frac{\mathrm{i} \sqrt{\pi}}{2}\right) \mathrm{e}^{-\frac{1}{4}}\left(-\frac{1}{2} \operatorname{erf}\left(\frac{\mathrm{i}}{2}\right)+\right. \\
& \left.\frac{1}{2} \operatorname{erf}\left(\frac{i}{2}(1+2 t)\right)+\operatorname{terf}\left(\frac{i}{2}(1+2 t)\right)-\operatorname{terf}\left(\frac{i}{2}\right)\right) \\
& a_{36}=-\frac{1}{4}+\frac{1}{4} e^{t+t^{2}}-\frac{1}{2} t e^{t+t^{2}} \\
& +\left(\frac{\mathrm{i} \sqrt{\pi}}{8}\right) \mathrm{e}^{-\frac{1}{4}}\left(\operatorname{erf}\left(\frac{\mathrm{i}}{2}\right)+4 \mathrm{t}^{2} \operatorname{erf}\left(\frac{\mathrm{i}}{2}\right)\right. \\
& -4 t^{2} \operatorname{erf}\left(\frac{i}{2}(1+2 t)\right)-\operatorname{erf}\left(\frac{i}{2}(1+2 t)\right)
\end{aligned}
$$

where, $\operatorname{erf}(\mathrm{x})$ is the error function. The rest of the components were obtained using the Maple package. Using the Maple package the inverse Fourier transform of Eq. 27 is:

$$
\begin{aligned}
& u_{0}(x, t)=e^{-x} \\
& u_{1}(x, t)=e^{-x}\left(1+\left(t+t^{2}\right)\right) \\
& u_{2}(x, t)=e^{-x}\left(1+t+\frac{3}{2} t^{2}+t^{3}+\frac{t^{4}}{2}\right) \\
& =e^{-x}\left(1+\left(t+t^{2}\right)+\frac{1}{2}\left(t+t^{2}\right)^{2}\right) \\
& u_{3}(x, t)=e^{-x}\left(1+t+\frac{3}{2} t^{2}+\frac{7}{6} t^{3}+t^{4}+\frac{t^{5}}{2}+\frac{t^{6}}{6}\right)= \\
& e^{-x}\left(1+\left(t+t^{2}\right)+\frac{1}{2}\left(t+t^{2}\right)^{2}+\frac{1}{6}\left(t+t^{2}\right)^{3}\right)
\end{aligned}
$$

and so on. The Taylor series expansion for $\mathrm{e}^{\mathrm{t}}$ is written as:

$e^{t}=\sum_{n=0}^{\infty} \frac{t^{n}}{n !}$

By substituting Eq. 41 into Eq. 40, thus Eq. 40 can ultimately be reduced to Eq. 42:

$$
\mathrm{u}_{\mathrm{o}}(\mathrm{x}, 0)=\mathrm{e}^{-\mathrm{x}} \mathrm{e}^{\mathrm{t}+\mathrm{t}^{2}}
$$

This is the exact solution of the problem, Eq. 35. In the following, Table 2, the trend of convergence of the results of $u_{0}(x, t)$ to $u_{s}(x, t)$ of the FTVIM solution of Eq. 35 towards the exact solution is shown. Table 2 shows the percentage of relative errors of the results of $\mathrm{u}_{0}(\mathrm{x}, \mathrm{t})$ to $u_{s}(x, t)$ of the FTVIM solution of Eq. 35 at each location along the $\mathrm{x}$ axis and at different times. The trend of very rapid convergence, the maximum relative error of less than $0.15 \%$, of the solution towards the exact solution is clearly shown.

Example 3: The Kolmogorov-Petrovskii-Piskunov (KPP) is obtained by taking $D=1, r=-\left(-1+4 x^{2}\right)$ in Eq. 24 as follow Eq. 43:

$$
\begin{aligned}
& \frac{\partial u}{\partial t}=\frac{\partial^{2} u}{\partial x^{2}}-\left(-1+4 x^{2}\right) u x \geq 0, t \geq 0 \\
& u(x, 0)=e^{-x^{2}}, u(0, t)=e^{-t}, u(0, t)=0
\end{aligned}
$$

By applying the Fourier transform to Eq. 43 we obtain the following Eq. 44:

$$
\frac{\partial \hat{\mathrm{u}}}{\partial \mathrm{t}}+i \omega \mathrm{e}^{-\mathrm{t}}+\left(\omega^{2}-1-4 \frac{\partial^{2}}{\partial \omega^{2}}\right) \hat{\mathrm{u}}=0
$$


Phy. Intl. 2 (1): 8-20, 2011

Table 2: Shows the percentage of relative errors of the results of $u_{0}(x, t)$ to $u_{s}(x, t)$ of the FTVIM solution of Eq. 35

\begin{tabular}{|c|c|c|c|c|}
\hline & & \multicolumn{3}{|c|}{ Percentage of relative error (\%RE) } \\
\hline & & $x=1$ & $\mathrm{x}=1.5$ & $x=2$ \\
\hline \multirow[t]{6}{*}{$\mathrm{t}=0.1$} & $\mathrm{u}_{0}(\mathrm{x}, \mathrm{t})$ & 0.1041658643 & 0.1041658644 & 0.1041658643 \\
\hline & $\mathrm{u}_{1}(\mathrm{x}, \mathrm{t})$ & 0.005624109412 & 0.00562410955 & 0.005624109412 \\
\hline & $\mathrm{u}_{2}(\mathrm{x}, \mathrm{t})$ & 0.0002043128913 & 0.00020431290 & 0.0002043128913 \\
\hline & $\mathrm{u}_{3}(\mathrm{x}, \mathrm{t})$ & 0.000005587317504 & 0.000005587466 & 0.000005587317504 \\
\hline & $\mathrm{u}_{4}(\mathrm{x}, \mathrm{t})$ & $1.227292766 \mathrm{e}-7$ & $1.22854411 \mathrm{e}-7$ & $1.227292766 \mathrm{e}-7$ \\
\hline & $\mathrm{u}_{\mathrm{s}}(\mathrm{x}, \mathrm{t})$ & $2.687502407 \mathrm{e}-9$ & 2.81039503 e- 9 & 2.687502407 e- 9 \\
\hline \multirow[t]{6}{*}{$\mathrm{t}=0.4$} & $\mathrm{u}_{0}(\mathrm{x}, \mathrm{t})$ & 0.4287909361 & 0.4287909360 & 0.4287909361 \\
\hline & $\mathrm{u}_{1}(\mathrm{x}, \mathrm{t})$ & 0.1089138602 & 0.1089138601 & 0.1089138602 \\
\hline & $\mathrm{u}_{2}(\mathrm{x}, \mathrm{t})$ & 0.01934827902 & 0.01934827890 & 0.01934827902 \\
\hline & $\mathrm{u}_{3}(\mathrm{x}, \mathrm{t})$ & 0.002629370713 & 0.002629370740 & 0.002629370713 \\
\hline & $\mathrm{u}_{4}(\mathrm{x}, \mathrm{t})$ & 0.0002887233335 & 0.0002887234084 & 0.0002887233335 \\
\hline & $u_{s}(x, t)$ & 0.00002657093203 & 0.00002657081515 & 0.00002657093203 \\
\hline \multirow[t]{6}{*}{$\mathrm{t}=0.7$} & $\mathrm{u}_{0}(\mathrm{x}, \mathrm{t})$ & 0.6957787359 & 0.6957787359 & 0.6957787359 \\
\hline & $\mathrm{u}_{1}(\mathrm{x}, \mathrm{t})$ & 0.3337554316 & 0.3337554317 & 0.3337554316 \\
\hline & $\mathrm{u}_{2}(\mathrm{x}, \mathrm{t})$ & 0.1183515656 & 0.1183515656 & 0.1183515656 \\
\hline & $\mathrm{u}_{3}(\mathrm{x}, \mathrm{t})$ & 0.03290803214 & 0.03290803214 & 0.03290803214 \\
\hline & $\mathrm{u}_{4}(\mathrm{x}, \mathrm{t})$ & 0.007488581039 & 0.007488581083 & 0.007488581039 \\
\hline & $\mathrm{u}_{\mathrm{s}}(\mathrm{x}, \mathrm{t})$ & 0.001438751495 & 0.001438751489 & 0.001438751495 \\
\hline
\end{tabular}

$$
\hat{\mathrm{u}}(\omega, 0)=\frac{\sqrt{\pi}}{2} \mathrm{e}^{-\frac{\omega^{2}}{4}} \operatorname{erf}\left(\frac{\mathrm{i} \omega}{2}\right)
$$

where, erf (x) is the error function. We construct a correction functional as Eq. 45:

$$
\begin{aligned}
& \hat{\mathrm{u}}_{\mathrm{n}+1}(\omega, \mathrm{t})=\hat{\mathrm{u}}_{\mathrm{n}}(\omega, \mathrm{t}) \\
& +\int_{0}^{\mathrm{t}} \lambda(\xi)\left(\frac{\partial \hat{\mathrm{u}}_{\mathrm{n}}}{\partial \xi}+i \omega \mathrm{e}^{-\xi}+\left(\omega^{2}-1-4 \frac{\partial^{2}}{\partial \omega^{2}}\right) \hat{\mathrm{u}}_{\mathrm{n}}\right) \mathrm{d} \xi
\end{aligned}
$$

Integrating by parts we get $\lambda(\xi)=-1$. Assuming $\hat{\mathrm{u}}_{0}(\omega, \mathrm{t})=\hat{\mathrm{u}}(\omega, 0)$ and substituting for the value of $\lambda(\xi)=$ -1, Eq. 45 can be rewritten as Eq. 46:

$$
\begin{aligned}
& \hat{\mathrm{u}}_{0}(\omega, \mathrm{t})=\frac{\sqrt{\pi}}{2} \mathrm{e}^{-\frac{\omega^{2}}{4}} \operatorname{erf}\left(\frac{i \omega}{2}\right) \\
& \hat{\mathrm{u}}_{1}(\omega, \mathrm{t})=\hat{\mathrm{u}}_{0}(\omega, \mathrm{t})-\int_{0}^{\mathrm{t}}\left(\frac{\partial \hat{\mathrm{u}}_{0}}{\partial \xi}+\mathrm{i} \omega \mathrm{e}^{-\xi}+\left(\omega^{2}-1-4 \frac{\partial^{2}}{\partial \omega^{2}}\right) \hat{\mathrm{u}}_{0}\right) \mathrm{d} \xi \\
& \hat{\mathrm{u}}_{2}(\omega, \mathrm{t})=\hat{\mathrm{u}}_{1}(\omega, \mathrm{t})-\int_{0}^{\mathrm{t}}\left(\frac{\partial \hat{\mathrm{u}}_{1}}{\partial \xi}+\mathrm{i} \omega \mathrm{e}^{-\xi}+\left(\omega^{2}-1-4 \frac{\partial^{2}}{\partial \omega^{2}}\right) \hat{\mathrm{u}}_{1}\right) \mathrm{d} \xi \\
& \hat{\mathrm{u}}_{3}(\omega, \mathrm{t})=\hat{\mathrm{u}}_{2}(\omega, \mathrm{t})-\int_{0}^{\mathrm{t}}\left(\frac{\partial \hat{\mathrm{u}}_{2}}{\partial \xi}+\mathrm{i} \omega \mathrm{e}^{-\xi}+\left(\omega^{2}-1-4 \frac{\partial^{2}}{\partial \omega^{2}}\right) \hat{\mathrm{u}}_{2}\right) \mathrm{d} \xi
\end{aligned}
$$

$$
\hat{\mathrm{u}}_{\mathrm{n}+1}(\omega, \mathrm{t})=\hat{\mathrm{u}}_{\mathrm{n}}(\omega, \mathrm{t})-\int_{0}^{\mathrm{t}}\left(\frac{\partial \hat{\mathrm{u}}_{\mathrm{n}}}{\partial \xi}+\mathrm{i} \omega \mathrm{e}^{-\xi}+\left(\omega^{2}-1-\frac{\partial^{2}}{\partial \omega^{2}}\right) \hat{\mathrm{u}}_{\mathrm{n}}\right) \mathrm{d} \xi
$$

And so on. By integrating the recursive approximate equations by parts, Eq. 46, we obtain the followings Eq. 47:

$$
\begin{aligned}
& \hat{u}_{0}(\omega, t)=\frac{\sqrt{\pi}}{2} e^{-\frac{\omega^{2}}{4}} \operatorname{erf}\left(\frac{i \omega}{2}\right) \\
& \hat{u}_{1}(\omega, t)=\frac{\sqrt{\pi}}{2} e^{-\frac{\omega^{2}}{4}} \operatorname{erf}\left(\frac{i \omega}{2}\right)(1-t)-i \omega\left(1-t-e^{-t}\right) \\
& \hat{u}_{2}(\omega, t)=\frac{\sqrt{\pi}}{2} e^{-\frac{\omega^{2}}{4}} \operatorname{erf}\left(\frac{i \omega}{2}\right)\left(1-t+\frac{t^{2}}{2}\right) \\
& -i \omega\left(\omega^{2}-1\right)\left(1-t+\frac{t^{2}}{2}-e^{-t}\right)-i \omega\left(1-t+\frac{t^{2}}{2}-e^{-t}\right) \\
& \hat{u}_{3}(\omega, t)=\frac{\sqrt{\pi}}{2} e^{-\frac{\omega^{2}}{4}} \operatorname{erf}\left(\frac{i \omega}{2}\right)\left(1-t+\frac{t^{2}}{2}-\frac{t^{3}}{6}\right) \\
& -i \omega\left(\left(\omega^{2}-1\right)^{2}-24\right)\left(1-t+\frac{t^{2}}{2}-\frac{t^{3}}{6}-e^{-t}\right) i \omega\left(\left(\omega^{2}-1\right)\right. \\
& \left(1-t+\frac{t^{2}}{2}-\frac{t^{3}}{6}-e^{-t}\right)-i \omega\left(1-t+\frac{t^{2}}{2}-\frac{t^{3}}{6}-e^{-t}\right)
\end{aligned}
$$

And so on. The rest of the components were obtained using the Maple package. Using the Maple package the inverse Fourier transform of Eq. 47 is:

$$
\mathrm{u}_{0}=\mathrm{e}^{-\mathrm{x}^{2}}, \mathrm{u}_{1}=\mathrm{e}^{-\mathrm{x}^{2}}(1-\mathrm{t})
$$


Phy. Intl. 2 (1): 8-20, 2011

Table 3: Shows the percentage of relative errors of the results of $\mathrm{u}_{0}(\mathrm{x}, \mathrm{t})$ to $\mathrm{u}_{\mathrm{s}}(\mathrm{x}, \mathrm{t})$ of the FTVIM solution of Eq. 43

\begin{tabular}{|c|c|c|c|c|}
\hline & & \multicolumn{3}{|c|}{ Percentage of relative error (\%RE) } \\
\hline & & $\mathrm{x}=1$ & $\mathrm{x}=1.5$ & $x=2$ \\
\hline \multirow[t]{6}{*}{$\mathrm{t}=0.1$} & $\mathrm{u}_{0}(\mathrm{x}, \mathrm{t})$ & 0.1051709181 & 0.1051709181 & 0.1051709181 \\
\hline & $\mathrm{u}_{1}(\mathrm{x}, \mathrm{t})$ & 0.005346173692 & 0.005346173731 & 0.005346173692 \\
\hline & $\mathrm{u}_{2}(\mathrm{x}, \mathrm{t})$ & 0.0001796808982 & 0.0001796808276 & 0.0001796808982 \\
\hline & $\mathrm{u}_{3}(\mathrm{x}, \mathrm{t})$ & 0.000004514291649 & 0.000004514352332 & 0.000004514291649 \\
\hline & $\mathrm{u}_{4}(\mathrm{x}, \mathrm{t})$ & $9.062401529 \mathrm{e}-8$ & $9.059532239 \mathrm{e}-8$ & $9.062401529 \mathrm{e}-8$ \\
\hline & $u_{s}(x, t)$ & $1.436722194 \mathrm{e}-9$ & $1.467979761 \mathrm{e}-9$ & $1.436722194 \mathrm{e}-9$ \\
\hline \multirow[t]{6}{*}{$\mathrm{t}=0.4$} & $\mathrm{u}_{0}(\mathrm{x}, \mathrm{t})$ & 0.4918246977 & 0.4918246978 & 0.4918246977 \\
\hline & $\mathrm{u}_{1}(\mathrm{x}, \mathrm{t})$ & 0.1049051814 & 0.1049051813 & 0.1049051814 \\
\hline & $\mathrm{u}_{2}(\mathrm{x}, \mathrm{t})$ & 0.01444079445 & 0.01444079451 & 0.01444079445 \\
\hline & $\mathrm{u}_{3}(\mathrm{x}, \mathrm{t})$ & 0.001472002375 & 0.001472002326 & 0.001472002375 \\
\hline & $\mathrm{u}_{4}(\mathrm{x}, \mathrm{t})$ & 0.0001192773519 & 0.0001192773575 & 0.0001192773519 \\
\hline & $\mathrm{u}_{\mathrm{s}}(\mathrm{x}, \mathrm{t})$ & 0.000008024972596 & 0.000008024915289 & 0.000008024972596 \\
\hline \multirow{6}{*}{$\mathrm{t}=0.7$} & $\mathrm{u}_{0}(\mathrm{x}, \mathrm{t})$ & 1.013752707 & 1.013752707 & 1.013752707 \\
\hline & $\mathrm{u}_{1}(\mathrm{x}, \mathrm{t})$ & 0.3958741878 & 0.3958741878 & 0.3958741878 \\
\hline & $\mathrm{u}_{2}(\mathrm{x}, \mathrm{t})$ & 0.09749522555 & 0.09749522557 & 0.09749522555 \\
\hline & $\mathrm{u}_{3}(\mathrm{x}, \mathrm{t})$ & 0.01762430429 & 0.01762430440 & 0.01762430429 \\
\hline & $\mathrm{u}_{4}(\mathrm{x}, \mathrm{t})$ & 0.002521613488 & 0.002521613516 & 0.002521613488 \\
\hline & $u_{a}(x, t)$ & 0.0002988149244 & 0.0002988150145 & 0.0002988149244 \\
\hline
\end{tabular}

$\mathrm{u}_{2}=\mathrm{e}^{-\mathrm{x}^{2}}\left(1-\mathrm{t}+\frac{\mathrm{t}^{2}}{2}\right)$

$u_{3}=e^{-x^{2}}\left(1-t+\frac{t^{2}}{2}-\frac{t^{3}}{6}\right)$

$$
\begin{gathered}
u(x, 0)=1-e^{-\frac{x}{\sqrt{2}}} \\
u(0, t)=1-e^{-\frac{t}{2}}, u_{x}(0, t)=\frac{1}{\sqrt{2}} e^{-\frac{t}{2}}
\end{gathered}
$$

By applying the Fourier transform to Eq. 51 we

And so on. The Taylor series expansion for $\mathrm{e}^{-\mathrm{t}}$ is written as:

$$
\mathrm{e}^{-\mathrm{t}}=\sum_{\mathrm{n}=0}^{\infty} \frac{(-1)^{\mathrm{n}} \mathrm{t}^{\mathrm{n}}}{\mathrm{n} !}
$$

Substituting Eq. 49 into Eq. 48, the closed form solution of Eq. 43 is given by Eq. 50:

$$
u(x, t)=e^{-\left(x^{2}+t\right)}
$$

This is the exact solution of the problem, Eq. 43. In the following, Table 3, we have shown the trend of convergence of the results of $u_{0}(x, t)$ to $u_{s}(x, t)$ of the FTVIM solutions of Eq. 43 towards the exact solution. Table 3 shows the percentage of relative errors of the results of $u_{0}(x, t)$ to $u_{s}(x, t)$ of the FTVIM solution of Eq. 43 at different locations along the $\mathrm{x}$ axis and at different times. The trend of very rapid convergence, the relative error of $0.03 \%$, of the solution towards the exact solution is clearly shown.

Example 4: Consider the following nonlinear differential equation, the nonlinear Cauchy reactiondiffusion as the forth case study problem:

$$
\frac{\partial u}{\partial t}=\frac{\partial}{\partial x}\left(u \frac{\partial u}{\partial x}\right)+u(1-u) x \geq 0, t \geq 0
$$

obtain as follow Eq. 52:

$$
\begin{aligned}
& \frac{\partial \hat{\mathrm{u}}}{\partial \mathrm{t}}-\hat{\mathrm{u}}+\left(1+\frac{\omega^{2}}{2}\right) \mathrm{F}\left\{\mathrm{u}^{2}\right\}+\frac{1}{\sqrt{2}} \mathrm{e}^{-\frac{\mathrm{t}}{2}}\left(1-\mathrm{e}^{-\frac{\mathrm{t}}{2}}\right)+\frac{\mathrm{i} \omega}{2}\left(1-\mathrm{e}^{-\frac{\mathrm{t}}{2}}\right)=0 \\
& \hat{\mathrm{u}}(\omega, 0)=\pi \delta(\omega)-\frac{\mathrm{i} \sqrt{2}}{\omega(2 \mathrm{i} \omega+\sqrt{2})}
\end{aligned}
$$

where, superscript on the dependent variable $\mathrm{u}$ indicates the Fourier transform, $F\left\{u^{2}\right\}$ is the Fourier transform of $u^{2}$ and $\delta(\omega)$ is the Dirac delta function. We construct a correction functional as:

$$
\begin{aligned}
& \hat{\mathrm{u}}_{\mathrm{n}+1}(\omega, \mathrm{t})=\hat{\mathrm{u}}_{\mathrm{n}}(\omega, \mathrm{t}) \\
& +\int_{0}^{\mathrm{t}}\left(\begin{array}{l}
\lambda(\xi) \frac{\partial \hat{\mathrm{u}}_{\mathrm{n}}}{\partial \xi}-\hat{\mathrm{u}}+\left(1+\frac{\omega^{2}}{2}\right) \mathrm{F}\left\{\mathrm{u}_{\mathrm{n}}^{2}\right\} \\
+\frac{1}{\sqrt{2}} \mathrm{e}^{-\frac{\xi}{2}}\left(1-\mathrm{e}^{-\frac{\xi}{2}}\right)+\frac{\mathrm{i} \omega}{2}\left(1-\mathrm{e}^{-\frac{\xi}{2}}\right)^{2} \mathrm{~d} \xi
\end{array}\right.
\end{aligned}
$$

Integrating by parts we get $\mathrm{d}(\xi)=-1$. Assuming $\hat{\mathrm{u}}_{0}(\omega, \mathrm{t})=\hat{\mathrm{u}}(\omega, 0)$ and substituting for the value of $\mathrm{d}(\xi)$ $=-1$, Eq. 53 can be rewritten as: 
Phy. Intl. 2 (1): 8-20, 2011

$$
\begin{gathered}
\hat{\mathrm{u}}_{0}(\omega, \mathrm{t})=\pi \delta(\omega)-\frac{\mathrm{i} \sqrt{2}}{\omega(2 \mathrm{i} \omega+\sqrt{2})} \\
\hat{\mathrm{u}}_{1}(\omega, \mathrm{t})=\hat{\mathrm{u}}_{0}(\omega, \mathrm{t}) \\
\int_{0}^{\mathrm{t}}\left(\frac{\partial \hat{\mathrm{u}}_{0}}{\partial \xi}-\hat{\mathrm{u}}_{0}+\left(1+\frac{\omega^{2}}{2}\right) \mathrm{F}\left\{\mathrm{u}_{0}^{2}\right\}+\right. \\
\frac{1}{\sqrt{2}} \mathrm{e}^{-\frac{\xi}{2}}\left(1-\mathrm{e}^{-\frac{\xi}{2}}\right)+\frac{\mathrm{i} \omega}{2}\left(1-\mathrm{e}^{-\frac{\xi}{2}}\right)^{2} \mathrm{~d} \xi
\end{gathered}
$$

$$
\mathrm{A}_{22}=-\left|\begin{array}{l}
14 \omega^{3}-\sqrt{2} \mathrm{i} \omega^{2}+6 \omega+\sqrt{2} \mathrm{i} \omega^{2} \mathrm{e}^{-\mathrm{t}}+ \\
\mathrm{t}^{2}+\sqrt{2} \mathrm{i} t \omega^{2}-2 \mathrm{t} \omega-8 \omega \mathrm{e}^{\frac{-t}{2}} \\
-2 \mathrm{i} \omega^{2} \sqrt{2} \ln \left(\mathrm{e}^{\frac{-t}{2}}\right)^{2}+2 \omega \mathrm{e}^{-\mathrm{t}} \\
-6 \omega^{3} \mathrm{e}^{\mathrm{t}}+2 \omega^{3} \mathrm{e}^{-\mathrm{t}}-16 \omega^{3} \mathrm{e}^{\frac{-t}{2}} \\
-4 \omega^{3} \ln \left(\mathrm{e}^{\frac{-t}{2}}\right)-2 \sqrt{2} \mathrm{it}+ \\
4 \pi \mathrm{i} \delta(\omega) \omega^{2} \mathrm{t}+2 \sqrt{2} \pi \delta(\omega) \omega \mathrm{t}
\end{array}\right|
$$

$$
\hat{\mathrm{u}}_{2}(\omega, \mathrm{t})=\hat{\mathrm{u}}_{1}(\omega, \mathrm{t})
$$

$$
-\int_{0}^{t}\left(\begin{array}{l}
\frac{\partial \hat{\mathrm{u}}_{1}}{\partial \xi}-\hat{\mathrm{u}}_{1}+\left(1+\frac{\omega^{2}}{2}\right) \mathrm{F}\left\{\mathrm{u}_{1}^{2}\right\}+ \\
\frac{1}{\sqrt{2}} \mathrm{e}^{-\frac{\xi}{2}}\left(1-\mathrm{e}^{-\frac{\xi}{2}}\right)+\frac{i \omega}{2}\left(1-\mathrm{e}^{-\frac{\xi}{2}}\right)^{2}
\end{array}\right) \mathrm{d} \xi
$$

$$
\begin{aligned}
& \hat{\mathrm{u}}_{3}(\omega, \mathrm{t})=\hat{\mathrm{u}}_{2}(\omega, \mathrm{t}) \\
& \int_{0}^{\mathrm{t}}\left(\begin{array}{l}
\frac{\partial \hat{\mathrm{u}}}{\partial \xi}-\hat{\mathrm{u}}+\left(1+\frac{\omega^{2}}{2}\right) \mathrm{F}\left\{\mathrm{u}_{2}^{2}\right\}+ \\
\frac{1}{\sqrt{2}} \mathrm{e}^{-\frac{\xi}{2}}\left(1-\mathrm{e}^{-\frac{\xi}{2}}\right)+\frac{i \omega}{2}\left(1-\mathrm{e}^{-\frac{\xi}{2}}\right)^{2}
\end{array}\right) \mathrm{d \xi}
\end{aligned}
$$

And so on. By integrating the recursive approximate equations by parts, Eq. 54, we obtain the followings:

$$
\begin{gathered}
\hat{\mathrm{u}}_{0}(\omega, \mathrm{t})=\pi \delta(\omega)-\frac{\mathrm{i} \sqrt{2}}{\omega(2 \mathrm{i} \omega+\sqrt{2})} \\
\hat{\mathrm{u}}_{1}(\omega, \mathrm{t})=\pi \delta(\omega)-\frac{\mathrm{i} \sqrt{2}}{\omega(2 \mathrm{i} \omega+\sqrt{2})} \\
+\frac{\mathrm{t}}{2 \mathrm{i} \omega+\sqrt{2}}-\frac{1}{\sqrt{2}}+\sqrt{2} \mathrm{e}^{-\frac{\mathrm{t}}{2}}-\frac{1}{\sqrt{2}} \mathrm{e}^{-\mathrm{t}} \\
-\frac{1}{2} \mathrm{i} \omega\left(-3-\mathrm{e}^{-\mathrm{t}}+4 \mathrm{e}^{-\frac{\mathrm{t}}{2}}-2 \ln \left(\mathrm{e}^{-\frac{\mathrm{t}}{2}}\right)\right) \\
\hat{\mathrm{u}}_{2}(\omega . \mathrm{t})=\pi \delta(\omega)-\frac{\mathrm{i} \sqrt{2}}{\omega(2 \mathrm{i} \omega+\sqrt{2})}+-\frac{1}{4} \frac{1 \omega(2 \mathrm{i} \omega+\sqrt{2})(\mathrm{i} \omega+\sqrt{2})}{\left.\mathrm{A}_{21}\right]+\frac{1}{2 \omega(2 \mathrm{i} \omega+\sqrt{2})}\left[\mathrm{A}_{22}\right]+\mathrm{A}_{33}} \\
\mathrm{~A}_{21}=\left[-8 \mathrm{it}+\frac{\mathrm{t}^{3}}{3}\left(2 \mathrm{i} \omega^{2}+\sqrt{2} \omega\right)+2 \sqrt{2} \omega \mathrm{t}^{2}-8 \pi \delta(\omega) \omega^{3} \mathrm{t}\right. \\
\left.+12 \sqrt{2} \pi \mathrm{i} \delta(\omega) \omega^{2} \mathrm{t}+8 \pi \delta(\omega) \omega \mathrm{t}\right]
\end{gathered}
$$

$$
\begin{aligned}
& \mathrm{A}_{23}=-\frac{1}{\sqrt{2}}+\sqrt{2} \mathrm{e}^{-\frac{\mathrm{t}}{2}}-\frac{1}{2} \mathrm{e}^{-\mathrm{t}}-\frac{1}{2} \mathrm{i} \omega \\
& \left(-3-\mathrm{e}^{-\mathrm{t}}+4 \mathrm{e}^{-\frac{\mathrm{t}}{2}}-2 \ln \left(\mathrm{e}^{-\frac{\mathrm{t}}{2}}\right)\right) \\
& \hat{\mathrm{u}}_{3}(\omega \cdot \mathrm{t})=\pi \delta(\omega)-\frac{\mathrm{i} \sqrt{2}}{\omega(2 \mathrm{i} \omega+\sqrt{2})}+ \\
& \frac{1}{64} \frac{1+\frac{\omega^{2}}{2} \omega(2 \mathrm{i} \omega+\sqrt{2}) \omega(\mathrm{i} \omega+\sqrt{2})}{\left.\mathrm{A}_{31}\right]+} \\
& \frac{1}{24 \omega(2 \mathrm{i} \omega+\sqrt{2})(\mathrm{i} \omega+\sqrt{2})}\left[\mathrm{A}_{32}\right]+\mathrm{A}_{33}
\end{aligned}
$$

$$
\mathrm{A}_{31}=\left[-128 \mathrm{it}+\frac{\mathrm{t}^{5}}{5}\left(2 \mathrm{i} \omega^{2}+\sqrt{2} \omega\right)+\frac{\mathrm{t}^{4}}{4}\right.
$$$$
\left(-16 i \omega^{2}-8 \sqrt{2} \omega\right)+\frac{t^{4}}{4}\left(-16 i \omega^{2}\right.
$$$$
-8 \sqrt{2} \omega)+\frac{32}{3}\left(\mathrm{i} \omega^{2} \mathrm{t}^{3}+32 \sqrt{2} \omega \mathrm{t}^{2}\right.
$$$$
\left.-128 \pi \delta(\omega) \omega^{3} \mathrm{t}+192 \sqrt{2} \pi \mathrm{i} \delta(\omega) \omega^{2} \mathrm{t}+128 \pi \delta(\omega) \omega \mathrm{t}\right]
$$

$$
\mathrm{A}_{32}=-\left[\begin{array}{l}
96 \sqrt{2} \omega \mathrm{e}^{\frac{-\mathrm{t}}{2}}-42 \sqrt{2} \omega^{3} \mathrm{t}^{2}+192 \sqrt{2} \omega^{3} \mathrm{e}^{\frac{-\mathrm{t}}{2}} \\
-2 \sqrt{2} \omega^{3} \mathrm{t}^{3} \\
+4 i \omega^{2} \mathrm{t}^{3}+72 \sqrt{2} \omega \mathrm{t}+961 \mathrm{i} \omega^{2} \mathrm{e}^{\frac{-\mathrm{t}}{2}} \\
-48 \sqrt{2} \omega^{3} \ln \left(\mathrm{e}^{\frac{-\mathrm{t}}{2}}\right)^{3}-\mathrm{i} \omega^{2} \mathrm{t}^{4}-\frac{\mathrm{i} \omega^{4} \mathrm{t}^{4}}{2} \\
+32 \mathrm{i} \omega^{2} \ln \left(\mathrm{e}^{\frac{-\mathrm{t}}{2}}\right)^{3}+192 \mathrm{i} \omega^{4} \mathrm{e}^{\frac{-t}{2}}-36 \mathrm{i} \omega^{4} \mathrm{t}^{2} \\
+96 \mathrm{i} \omega^{4} \mathrm{t}+48 \mathrm{i} \omega^{2} \mathrm{t}-32 \mathrm{i} \omega^{4} \ln \left(\mathrm{e}^{\frac{-\mathrm{t}}{2}}\right)^{3} \\
-\frac{1}{\sqrt{2}} \omega \mathrm{t}^{4}+96 \sqrt{2} \omega^{3} \mathrm{t}-\frac{\sqrt{2} \omega^{3} \mathrm{t}^{4}}{4}-48 \mathrm{it} \\
-192 \sqrt{2} \omega^{3}-96 \mathrm{i} \omega^{2}+72 \sqrt{2} \pi \mathrm{i} \delta(\omega) \omega^{2} \mathrm{t} \\
-48 \pi \delta(\omega) \omega^{3} \mathrm{t}+48 \pi \delta(\omega) \omega \mathrm{t}-192 \mathrm{i} \omega^{4}
\end{array} \mid\right.
$$


Phy. Intl. 2 (1): 8-20, 2011

Table 4: shows: the percentage of relative errors of the results of $u_{0}(x, t)$ to $u_{s}(x, t)$ of the FTVIM solution of Eq. 51

\begin{tabular}{|c|c|c|c|c|}
\hline & & \multicolumn{3}{|c|}{ Percentage of Relative Error (\%RE) } \\
\hline & & $\mathrm{x}=1$ & $\mathrm{x}=1.5$ & $x=2$ \\
\hline \multirow[t]{6}{*}{$\mathrm{t}=0.1$} & $\mathrm{u}_{0}(\mathrm{x}, \mathrm{t})$ & 0.04528854092 & 0.02517778762 & 0.01542386214 \\
\hline & $\mathrm{u}_{1}(\mathrm{x}, \mathrm{t})$ & 0.001141648184 & 0.0006346899044 & 0.0003888097652 \\
\hline & $\mathrm{u}_{2}(\mathrm{x}, \mathrm{t})$ & 0.0000191066097 & 0.00001062209702 & 0.000006507009708 \\
\hline & $\mathrm{u}_{3}(\mathrm{x}, \mathrm{t})$ & $2.389927040 \mathrm{e}-7$ & $1.330036012 \mathrm{e}-7$ & 8.156201446 e- 8 \\
\hline & $\mathrm{u}_{4}(\mathrm{x}, \mathrm{t})$ & 2.448309812 e-9 & $1.341964586 \mathrm{e}-9$ & 7.804977460 e-10 \\
\hline & $\mathrm{u}_{\mathrm{s}}(\mathrm{x}, \mathrm{t})$ & $5.64994572 \mathrm{e}-10$ & $2.982143524 \mathrm{e}-10$ & $1.300829577 \mathrm{e}-10$ \\
\hline \multirow[t]{6}{*}{$\mathrm{t}=0.4$} & $\mathrm{u}_{0}(\mathrm{x}, \mathrm{t})$ & 0.1498855718 & 0.08758887901 & 0.05502145006 \\
\hline & $\mathrm{u}_{1}(\mathrm{x}, \mathrm{t})$ & 0.01548784334 & 0.009050656766 & 0.005685427655 \\
\hline & $\mathrm{u}_{2}(\mathrm{x}, \mathrm{t})$ & 0.001049498124 & 0.0006132967980 & 0.0003852601297 \\
\hline & $\mathrm{u}_{3}(\mathrm{x}, \mathrm{t})$ & 0.00005299093855 & 0.00003096660541 & 0.00001945233099 \\
\hline & $\mathrm{u}_{4}(\mathrm{x}, \mathrm{t})$ & 0.000002133791266 & 0.000001246697341 & 7.834418689 e-7 \\
\hline & $\mathrm{u}_{\mathrm{s}}(\mathrm{x}, \mathrm{t})$ & 7.143941207 e-8 & $4.186826399 \mathrm{e}-8$ & $2.609392042 \mathrm{e}-8$ \\
\hline \multirow{6}{*}{$\mathrm{t}=0.7$} & $\mathrm{u}_{0}(\mathrm{x}, \mathrm{t})$ & 0.2231418390 & 0.1352415250 & 0.08663826135 \\
\hline & $\mathrm{u}_{1}(\mathrm{x}, \mathrm{t})$ & 0.04132309083 & 0.02504504695 & 0.01604432753 \\
\hline & $\mathrm{u}_{2}(\mathrm{x}, \mathrm{t})$ & 0.004958271810 & 0.003005103159 & 0.001925125530 \\
\hline & $\mathrm{u}_{3}(\mathrm{x}, \mathrm{t})$ & 0.0004412202143 & 0.0002674141922 & 0.0001713105799 \\
\hline & $\mathrm{u}_{4}(\mathrm{x}, \mathrm{t})$ & 0.00003123500257 & 0.00001893090247 & 0.00001212750132 \\
\hline & $\mathrm{u}_{\mathrm{s}}(\mathrm{x}, \mathrm{t})$ & 0.000001836667987 & 0.000001113201244 & $7.130629992 \mathrm{e}-7$ \\
\hline
\end{tabular}

$\mathrm{A}_{33}=-\frac{1}{\sqrt{2}}+\sqrt{2} \mathrm{e}^{-\frac{\mathrm{t}}{2}}-\frac{1}{2} \mathrm{e}^{-\mathrm{t}}-\frac{1}{2}$

$i \omega\left(-3-e^{-t}+4 e^{-\frac{t}{2}}-2 \ln \left(e^{-\frac{t}{2}}\right)\right)$

The rest of the components were obtained using the Maple package. Using the Maple package the inverse Fourier transform of Eq. 55 is:

$$
\begin{aligned}
& u_{0}=1-e^{-\frac{x}{\sqrt{2}}}, \\
& u_{1}=1-e^{-\frac{x}{\sqrt{2}}}+\frac{1}{2} e^{-\frac{x}{\sqrt{2}}} t, \\
& u_{2}=1-e^{-\frac{x}{\sqrt{2}}}+\frac{1}{2} e^{-\frac{x}{\sqrt{2}}} t-\frac{1}{8} e^{-\frac{x}{\sqrt{2}}} t^{2} \\
& u_{3}=1-e^{-\frac{x}{\sqrt{2}}}+\frac{1}{2} e^{-\frac{x}{\sqrt{2}}} t-\frac{1}{8} e^{-\frac{x}{\sqrt{2}}} t^{2}+\frac{1}{48} e^{-\frac{x}{\sqrt{2}}} t^{3},
\end{aligned}
$$

And so on. The Taylor series expansion for $u(x, t)=1-e^{-\frac{x+\frac{t}{\sqrt{2}}}{\sqrt{2}}}$ is written as:

$$
\begin{aligned}
& 1-e^{-\frac{x+\frac{t}{\sqrt{2}}}{\sqrt{2}}}=1-\frac{1}{2} e^{-\frac{x}{\sqrt{2}}} t+\frac{1}{2} e^{-\frac{x}{\sqrt{2}}} t \\
& -\frac{1}{8} e^{-\frac{x}{\sqrt{2}}} t^{2}+\frac{1}{48} e^{-\frac{x}{\sqrt{2}}} t^{3}+\ldots
\end{aligned}
$$

Substituting Eq. 57 into Eq. 56, the closed form solution of Eq. 51 is given by Eq. 58 : $u(x, t)=1-e^{-\frac{x+\frac{t}{\sqrt{2}}}{\sqrt{2}}}$

This is the exact solution of the problem, Eq. 51. In the following, Table 4, we have shown the trend of convergence of the results of $\mathrm{u}_{0}(\mathrm{x}, \mathrm{t})$ to $\mathrm{u}_{\mathrm{a}}(\mathrm{x}, \mathrm{t})$ of the FTVIM solutions of Eq. 51 towards the exact solution. Table 4 shows the percentage of relative errors of the results of $\mathrm{u}_{0}(\mathrm{x}, \mathrm{t})$ to $\mathrm{u}_{\mathrm{a}}(\mathrm{x}, \mathrm{t})$ of the FTVIM solution of Eq. 51 at different locations along the $\mathrm{x}$ axis and at different times. The trend of very rapid convergence, the relative error of $0.0002 \%$, of the solution towards the exact solution is clearly shown.

\section{DISCUSSION}

Case study: The Cauchy reaction-diffusion equation expresses the mathematical models of the influence of the chemical reaction which the substances are transformed into each other and the diffusion which the substances are dispersed over a surface in space. This equation has wide applications in chemical engineering, biology, geology, ecology and physics.

We solve four one-dimensional transient and nonhomogeneous linear and nonlinear partial differential equations, the Cauchy reaction-diffusion problem, to demonstrate the effectiveness and the validity of the present method, FTVIM, in the entire range of problem domain. The validity and effectiveness of the new method are shown in Table 1-4 by solving four linear and nonlinear non-homogenous differential equations of Cauchy reaction-diffusion problem. Moreover, the very rapid convergence to the exact solutions is verified mathematically. The very rapid convergence towards 
Phy. Intl. 2 (1): 8-20, 2011

the exact solutions shown in tsbles 1-4 using the new method, FTVIM, indicates that the amount of computational work is much less than the computational work required for both the previous VIMs and the modified VIMs. Ultimately, the deficiency of the previous VIMs caused by unsatisfied boundary conditions is overcome by the new method (FTVIM) where, the solution is shown to be valid in the entire range of problem domain.

\section{CONCLUSION}

In this study a new effective modification to the VIM, the Fourier transform variational iteration method (FTVIM), is proposed. The new modification to the VIM is the combination of the Fourier transform and variational iteration method. The validity and effectiveness of the new method is shown by solving four linear and nonlinear non-homogenous differential equations of Cauchy reaction-diffusion problem and the very rapid convergence to the exact solutions is verified mathematically. The very rapid convergence towards the exact solutions using the new method, FTVIM, indicates that the amount of computational work is much less than the computational work required for both the previous VIMs and the modified VIMs. Moreover, the deficiency of the previous VIMs caused by unsatisfied boundary conditions is overcome by the new method (FTVIM) where, the solution is shown to be valid in the entire range of problem domain. Therefore, it is concluded that the FTVIM can be considered as a powerful and efficient tool in obtaining the accurate solutions to both linear and nonlinear partial differential equations as well as other effective numerical methods.

\section{REFERENCES}

Afshari, H.H., A.B. Novinzadeh and J. Roshanian, 2009. Determination of nonlinear optimal feedback law for satellite injection problem using neighboring optimal control. Am. J. Applied Sci., 6: 430-438. DOI: 10.3844/ajassp.2009.430.438

Akbarzade, M. and J. Langari, 2011. Application of homotopy-perturbation method and variational iteration method to three dimensional diffusion problem. Int. J. Math. Anal., 5: 871-880.

Aruchunan, E. and J. Sulaiman, 2010. Numerical solution of second-order linear fredholm integrodifferential equation using generalized minimal residual method. Am. J. Applied Sci., 7: 780-783. DOI: 10.3844/ajassp.2010.780.783
Aslanov, A., 2011. Analyzing homotopy perturbation method for solving fourth-order boundary value problem. Math. Problems Eng. DOI: $10.1155 / 2011 / 296796$

Chen, $\mathrm{X}$. and L. Wang, 2010. The variational iteration method for solving a neutral functional-differential equation with proportional delays. Comput. Math. Appli., 59: 2696-2702. $\quad$ DOI: 10.1016/j.camwa.2010.01.037

Gepreel, K.A., 2011. The homotopy perturbation method applied to the nonlinear fractional Kolmogorov-Petrovskii-Piskunov equations. Applied Math. Lett., 24: 1428-1434. DOI: 10.1016/j.aml.2011.03.025

Gupta, P.K. and M. Singh, 2011. Homotopy perturbation method for fractional FornbergWhitham equation. Comput. Math. Appli., 61: 250254. DOI: 10.1016/j.camwa.2010.10.045

He, J.H., 2009. An elementary introduction to the homotopy perturbation method. Comput. Math. Appli., $\quad$ 57: 410-412. $\quad$ DOI: 10.1016/j.camwa.2008.06.003

He, J.H., G.C. Wu and F. Austin, 2010. The variational iteration method which should be followed. Nonl. Sci. Lett., A 1: 1-30.

Kasozi, J., F. Mayambala and C.W. Mahera, 2011. Dividend maximization in the cramer-lundberg model using homotopy analysis method. J. Math. Stat., 7: 61-67. DOI: 10.3844/jmssp.2011.61.67

Madani, M., M. Fathizadeh, Y. Khan and A. Yildirim, 2011. On the coupling of the homotopy perturbation method and Laplace transformation. Math. Comput. Model., 53: 1937-1945. DOI: 10.1016/j.mcm.2011.01.023

Odibat, Z.M., 2010. A study on the convergence of variational iteration method. Math. Comput. Model., $\quad$ 51: 1181-1192. $\quad$ DOI: 10.1016/j.mcm.2009.12.034

Othman, M.I.A., A.M.S. Mahdy and R.M. Farouk, 2010. Numerical solution of 12th order boundary value problems by using Homotopy Perturbation Method. J. Math. Comput. Sci., 1: 14-27.

Shakeri, S., R. Saadati, S.M. Vaezpour and J. Vahidi, 2009. Variational iteration method for solving integral equations. J. Applied Sci., 9: 799-800.

Shang, X. and D. Han, 2010 Application of the variational iteration method for solving nth-order integro-differential equations. J. Comput. Applied Math., 234: 1442-1447. $\quad$ DOI: 10.1016/j.cam.2010.02.020

Turkyilmazoglu, M., 2011. An optimal variational iteration method. Applied Math. Lett., 24: 762-765. DOI: $10.1016 /$ j.aml.2010.12.032 
Phy. Intl. 2 (1): 8-20, 2011

Wazwaz, A.M., 2009. Partial Differential Equations and Solitary Waves Theory. 1st Edn., Higher Education Press, Beijing, Springer Dordrecht Heidelberg, London, New York, ISBN: 9783642002502, pp: 741.

Zhao, Y. and A. Xiao, 2010. Variational iteration method for singular perturbation initial value problems. Comput. Phys. Commun., 181: 947-956. DOI: $10.1016 /$ j.cpc.2010.01.007
Zhou, X.W. and L. Yao, 2010. The variational iteration method for Cauchy problems. Comput. Math. Appli., $\quad 60$ : 756-760. $\quad$ DOI: 10.1016/j.camwa.2010.05.022 